\title{
Perfil de una Muestra de Estudiantes del Instituto Politécnico Nacional (IPN), México
}

\author{
Yasmín I. Jiménez, Josefina Hernández y José D. Ortega \\ Instituto Politécnico Nacional, Escuela Superior de Cómputo, Depto. de Ingeniería en Sistemas \\ Computacionales, Av. Juan de Dios Bátiz s/n esq. Av. Miguel Othón de Mendizabal. Colonia Lindavista. \\ Demarcación Territorial: Gustavo A. Madero. C. P. 07738, México. (e-mail yjimenezg@ipn.mx; \\ johernandezj@ipn.mx; david82d@hotmail.com)
}

Recibido Mar. 6, 2017; Aceptado May. 3, 2017; Versión final Jun. 28, 2017, Publicado Oct. 2017

\section{Resumen}

El objetivo de la investigación fue describir el perfil de una muestra de estudiantes del nivel superior del Instituto Politécnico Nacional (IPN, México) con base en sus necesidades de educación y sus expectativas sobre la educación superior y la práctica docente. Con el imperativo de la transformación del Sistema de Educación Superior para el siglo XXI, teniendo la innovación como eje, y con el objetivo de elevar la calidad educativa, los docentes se encuentran ante el reto de innovar sus prácticas con base en las necesidades reales de los estudiantes, tema de este estudio. Con un estudio exploratorio-descriptivo se consultó a 497 estudiantes para desarrollar un perfil. Los resultados muestran estudiantes permanentemente conectados a la red, con orientación hacia aprendizajes prácticos y concretos, alta autoestima y convencidos de tener éxito académico.

Palabras clave: perfil del estudiante; educación superior; investigación educativa; hábitos de estudio; enseñanza - aprendizaje

\section{Profile of a Sample of Students from the National Polytechnic Institute (IPN), México}

\begin{abstract}
The goal of this investigation is to describe the social profile of a sample of higher education students from the National Polytechnic Institute (IPN, Mexico), based on their education needs as well as on their expectations about their learning and teacher practices. As a consequence of the transformation of the Education System for the XXI century, which has innovation as its axis, and with the objective of raising the quality of education, teachers face the challenge of innovating their practices to fulfill student's real needs. With an exploratory-descriptive study, 497 students were surveyed in order to develop the profile. Results show that the students are permanently connected to the web, have an orientation towards practical and concrete knowledge, possess high self-esteem and they are certain about their academic success.
\end{abstract}

Keywords: student profile; higher education; education research; studying habits; teaching-learning process 


\section{INTRODUCCIÓN}

La innovación educativa tiene como meta lograr una educación de calidad para todos los estudiantes (UNESCO, 2007) orientando la enseñanza hacia nuevas formas de aprendizaje y una nueva comprensión de la enseñanza (Gimeno y Pérez, 2005; Carreras y Perrenoud, 2008; Zabalza, 2002). Un análisis amplio de la calidad educativa lo podemos encontrar en el documento de la Organización de las Naciones Unidas para la Educación, la Ciencia y la Cultura (1998) que establece como pilares de la educación el saber: ser, conocer, hacer y convivir; que entre otras implicaciones abarcan dos conceptos importantes para las instituciones educativas: la formación integral y el aprendizaje permanente.

Dentro de este contexto, la Tercera Reforma de la Educación Superior en América Latina (TRESAL) fue impulsada por la redefinición de los espacios de aprendizaje, el desarrollo de nuevas áreas de conocimiento, la contracción de recursos del gobierno, la rendición de cuentas, la acreditación de la calidad y la innovación de las prácticas docentes, entre otras (UNESCO, 2003; Rama, 2006; Marchesi, 2009). La respuesta de los docentes a la necesidad de innovar las prácticas educativas para mejorar los procesos de enseñanza se han basado, principalmente, en el uso intensivo de las tecnologías de la información. Estas innovaciones a pesar de producir cierto impacto sobre el aprendizaje de las y los estudiantes no han logrado aumentar la calidad educativa (UNESCO, 2007).

Principalmente el estudio de la problemática de la baja calidad de los aprendizajes se ha enfocado en el análisis de la práctica docente y del por qué ésta no presenta evidencia de una renovada enseñanza. Resultados de diversas investigaciones marcan como posibles causas, entre otras, las siguientes: (i) renuencia de los cuerpos académicos a adoptar las reformas educativas porque éstas carecen de un soporte epistemológico - pedagógico (Gimeno y Pérez, 2005); (ii) los profesores en servicio no comprenden todavía qué implica llevar a la práctica una enseñanza basada en competencias (Prawat, 1992; Windschitl, 2002) y que el mayor logro ha sido una enseñanza "en transición" respecto al espíritu de la reforma (Beca et al., 2006; Zembylas, 2010); (iii) los programas de desarrollo profesional de profesores no han logrado incidir en una nueva práctica docente (Cano, 2008; Gorodokin, 2005; Imbernón, 2000); y (iv) la reforma impone sobre el docente nuevas competencias difíciles de desarrollar y los docentes van avanzando poco a poco (Carreras y Perrenoud, 2008; Zabala y Arnau, 2007; Jiménez, et al., 2014).

Es importante enfatizar que los cambios a la práctica educativa han sido muchos y de diferente magnitud. Dubet (2004) señala que con esta reforma, el modelo de formación docente ha sido ampliamente desestabilizado y la relación entre modelo educativo y práctica pedagógica se ha transformado en un problema porque los marcos de análisis han cambiado radicalmente. Para ejemplificar lo anterior, baste comentar que hasta finales del siglo XX se habían implementado dos modelos educativos: el centrado en la enseñanza y el centrado en el aprendizaje, y que para inicio del siglo XXI se cambió una vez más el modelo educativo a uno centrado en el desempeño del estudiante que se dirige a los resultados del aprendizaje; al desarrollo del pensamiento crítico y a la resolución de problemas (Ruiz, 2009; Díaz-Barriga y Hernández, 2002; Retana, 2011). A cada uno de estos modelos corresponden diferentes prácticas educativas (tabla 1), y por lo tanto existe diferencia en las concepciones: del aprendizaje del alumno, de lo que debe hacer y ser un docente para enseñar, y de lo que es un alumno.

Díaz-Barriga y Hernández (2002) afirman que el aprendizaje, aunque de carácter individual y endógeno, se sitúa en el plano social y la experiencia compartida siendo el docente el encargado de mediar y organizar el encuentro de los estudiantes con el conocimiento. Por otro lado, se ha documentado ampliamente que el aprendizaje de los estudiantes universitarios se ve influido por factores de carácter interno o contextual que los lleva a tener un enfoque profundo o superficial hacia el aprendizaje (Monroy y Hernández, 2014).

Dentro de los factores de carácter interno que coadyuvan a que los estudiantes tengan un enfoque profundo por el aprendizaje, extracción de significados y búsqueda de conexiones con los conocimientos previos, se encuentran: la motivación intrínseca, los hábitos de estudio, el autoconcepto, las expectativas académicas y la actitud de éxito. Por el contrario, los factores de carácter externo relacionados con un enfoque superficial del aprendizaje, memorización y reproducción de los contenidos, mayormente hacen referencia al miedo al fracaso y a una motivación extrínseca de los estudiantes (Baerten, et al. 2010; Cornejo y Redondo, 2007). Por lo tanto, en el aula interactúan estudiantes con diferentes expectativas y estilos de aprendizaje. El estilo de aprendizaje es un constructo que incluye rasgos metacognitivos, cognitivos y afectivos que sirven como indicadores, relativamente estables, de cómo los estudiantes perciben las interacciones de enseñanza aprendizaje (Alonso y Gallego, 2004; Vermunt, 1996). Vermunt manifiesta que dicho constructo se basa en que el aprendizaje es un proceso activo que comprende cinco componentes: 1) el procesamiento cognitivo; 2) la regulación del aprendizaje de los contenidos; 3) los procesos afectivos o las actitudes; 4) las preferencias del tipo de actividades de aprendizaje y 5) el enfoque de aprendizaje. Para este investigador, existen cuatro estilos de aprendizaje (Tabla 2 ) que contemplan dichos componentes. 
Tabla 1: Contrastación de las prácticas educativas en los modelos educativos (Datos tomados de Gimeno y Pérez, 2005; Argudín, 2005; Morán, 2008; Fullan, 2015)

\begin{tabular}{|c|c|c|c|c|c|}
\hline \multirow{2}{*}{$\begin{array}{c}\text { Modelos } \\
\text { educativos }\end{array}$} & \multicolumn{2}{|c|}{ Centrado en la enseñanza } & \multicolumn{2}{|c|}{ Centrado en el aprendizaje } & \multirow{2}{*}{$\begin{array}{l}\text { Centrado en el } \\
\text { desempeño }\end{array}$} \\
\hline & $\begin{array}{l}\text { Presagio- } \\
\text { producto }\end{array}$ & Proceso-producto & Mediacional & Ecológico & \\
\hline $\begin{array}{l}\text { Proceso } \\
\text { enseñanza- } \\
\text { aprendizaje } \\
\text { (e-a) }\end{array}$ & $\begin{array}{l}\text { El proceso } \\
\text { depende de las: } \\
\text { Experiencias } \\
\text { formativas del } \\
\text { docente } \\
\text { Experiencias } \\
\text { profesionales de } \\
\text { los docentes } \\
\text { Comportamien- } \\
\text { to del docente } \\
\text { en el aula } \\
\text { El aprendizaje } \\
\text { es dirigido }\end{array}$ & $\begin{array}{l}\text { Resulta del } \\
\text { comportamiento } \\
\text { del profesor y del } \\
\text { rendimiento del } \\
\text { alumno } \\
\text { Se trabaja } \\
\text { buscando } \\
\text { conductas } \\
\text { observables en los } \\
\text { alumnos } \\
\text { El aprendizaje es } \\
\text { un acto reflejo de } \\
\text { la enseñanza }\end{array}$ & $\begin{array}{l}\text { Existe una } \\
\text { influencia mutua } \\
\text { entre el profesor y } \\
\text { el alumno } \\
\text { Tanto profesor } \\
\text { como alumno } \\
\text { construyen su } \\
\text { propio } \\
\text { conocimiento a } \\
\text { través de } \\
\text { procesos } \\
\text { mentales }\end{array}$ & $\begin{array}{l}\text { Existe una } \\
\text { influencia } \\
\text { dialéctica entre } \\
\text { profesor- } \\
\text { alumnos- } \\
\text { procesos } \\
\text { cognitivos } \\
\text { La vida áulica se } \\
\text { define como un } \\
\text { conjunto de } \\
\text { intercambios } \\
\text { socioculturales }\end{array}$ & $\begin{array}{l}\text { La integración del } \\
\text { conocimiento, } \\
\text { procesos } \\
\text { cognoscitivos, } \\
\text { habilidades, valores } \\
\text { y actitudes ante } \\
\text { problemas } \\
\text { contextualizados } \\
\text { La enseñanza } \\
\text { coadyuva al } \\
\text { desarrollo del } \\
\text { pensamiento crítico } \\
\text { del estudiante }\end{array}$ \\
\hline $\begin{array}{l}\text { Rol del } \\
\text { docente }\end{array}$ & $\begin{array}{l}\text { Responsable } \\
\text { del éxito o } \\
\text { fracaso de los } \\
\text { alumnos } \\
\text { Domina las } \\
\text { características } \\
\text { de un profesor } \\
\text { eficaz }\end{array}$ & $\begin{array}{l}\text { Protagonista del } \\
\text { aula que repite } \\
\text { comportamientos } \\
\text { productores de } \\
\text { resultados } \\
\text { positivos } \\
\text { La enseñanza } \\
\text { provoca la } \\
\text { respuesta } \\
\text { deseada en el } \\
\text { aprendizaje }\end{array}$ & $\begin{array}{l}\text { Sujeto reflexivo, } \\
\text { activo } \\
\text { Interpreta y } \\
\text { diagnostica cada } \\
\text { situación en el } \\
\text { aula } \\
\text { Desarrolla } \\
\text { procesos de } \\
\text { socialización y } \\
\text { pensamiento } \\
\text { pedagógico }\end{array}$ & $\begin{array}{l}\text { Considera los } \\
\text { contextos físicos } \\
\text { y psicosociales; } \\
\text { trabaja bajo una } \\
\text { perspectiva } \\
\text { sistémica } \\
\text { Reflexiona } \\
\text { constantemente } \\
\text { sobre su } \\
\text { desempeño }\end{array}$ & $\begin{array}{l}\text { Concibe el currículo } \\
\text { como un proyecto } \\
\text { de actividades para } \\
\text { que los estudiantes } \\
\text { puedan construir } \\
\text { competencias y a la } \\
\text { evaluación como un } \\
\text { instrumento de } \\
\text { aprendizaje de } \\
\text { saberes, destrezas, } \\
\text { actitudes y valores }\end{array}$ \\
\hline $\begin{array}{l}\text { Rol del } \\
\text { alumno }\end{array}$ & $\begin{array}{l}\text { Persona } \\
\text { dependiente } \\
\text { Rol pasivo en } \\
\text { su aprendizaje, } \\
\text { predomina la } \\
\text { memorización }\end{array}$ & $\begin{array}{l}\text { Recibe los } \\
\text { estímulos } \\
\text { aplicados por el } \\
\text { profesor y } \\
\text { reacciona a ellos }\end{array}$ & $\begin{array}{l}\text { Actor principal } \\
\text { Acomoda la } \\
\text { información con la } \\
\text { pre-existente } \\
\text { Responsable de } \\
\text { su aprendizaje }\end{array}$ & $\begin{array}{l}\text { Procesa } \\
\text { activamente la } \\
\text { información } \\
\text { Manifiesta y } \\
\text { demanda } \\
\text { aprendizaje }\end{array}$ & $\begin{array}{l}\text { Persona } \\
\text { comprometida con } \\
\text { la construcción de } \\
\text { sus competencias a } \\
\text { lo largo de vida }\end{array}$ \\
\hline
\end{tabular}

Díaz-Barriga (2006) manifiesta que no existe una sola vía para lograr el aprendizaje de los estudiantes, pero para lograrlo los docentes deberán reflexionar metódicamente sobre las características, creencias y carencias de los estudiantes; asimismo Pedró (2006) subraya que las mejores innovaciones deberán centrarse en nuevas propuestas que permitan acomodar los procesos y las actividades de aprendizaje con base en los cambios cognitivos y de comunicación que presentan los estudiantes. Adicionalmente, habría que reflexionar sobre lo que los estudiantes esperan de sus docentes y sus hábitos de estudio por ser factores determinantes del rendimiento académico (Osuna y Luna, 2008; Díaz-Barriga y Hernández 2002). Dentro de los rasgos característicos de las profesiones se encuentran cuatro competencias: 1) cognitivas y técnicas 2) sociales 3) éticas y 4) afectivas (Osuna y Luna, 2008) dentro de las cuales los estudiantes podrían otorgar una diferente valoración a cada una de ellas para conducir con éxito el proceso de enseñanza; y los hábitos de estudio se refieren a las diferentes acciones que emprenden las estudiantes, de manera constante, encaminadas a mejorar su desempeño académico; incluyen planeación y organización de tiempos y espacios para el estudio y métodos de estudio (Covey, 2012).

La Organización para la Cooperación y el Desarrollo Económico (OCDE) define como New Milennium Learners (NML) a las generaciones que nacieron a partir de los años 80 y que crecieron en un contexto donde las tecnologías digitales forman parte la vida cotidiana, de tal manera que la mayor parte de sus actividades están mediatizadas por dichas tecnologías (Pedró, 2006). 
Tabla 2: Componentes de los estilos de aprendizaje (Adaptada de Vermunt, 1996)

\begin{tabular}{|c|c|c|c|c|}
\hline \multirow[b]{2}{*}{ Componentes } & \multicolumn{4}{|c|}{ Estilos de aprendizaje } \\
\hline & No dirigido & $\begin{array}{l}\text { Dirigido a la } \\
\text { reproducción }\end{array}$ & Dirigido al significado & Dirigido a la aplicación \\
\hline $\begin{array}{l}\text { Procesamiento } \\
\text { cognitivo }\end{array}$ & Sin procesamiento & $\begin{array}{l}\text { Procesamiento por } \\
\text { etapas }\end{array}$ & $\begin{array}{l}\text { Procesamiento } \\
\text { profundo }\end{array}$ & Procesamiento concreto \\
\hline $\begin{array}{l}\text { Regulación del } \\
\text { aprendizaje }\end{array}$ & Sin regulación & Regulación externa & Autorregulación & $\begin{array}{l}\text { Regulación interna y } \\
\text { externa }\end{array}$ \\
\hline $\begin{array}{l}\text { Procesos } \\
\text { afectivos }\end{array}$ & $\begin{array}{l}\text { Baja autoestima } \\
\text { Temor al fracaso }\end{array}$ & $\begin{array}{l}\text { Baja autoestima } \\
\text { Temor al examen }\end{array}$ & Alta autoestima & Alta autoestima \\
\hline $\begin{array}{l}\text { Modelo mental } \\
\text { de aprendizaje }\end{array}$ & $\begin{array}{l}\text { Prefieren trabajo } \\
\text { cooperativo } \\
\text { El conocimiento se } \\
\text { absorbe }\end{array}$ & $\begin{array}{l}\text { Prefieren trabajo } \\
\text { cooperativo } \\
\text { El conocimiento } \\
\text { ingresa como } \\
\text { información }\end{array}$ & $\begin{array}{l}\text { Prefieren trabajo } \\
\text { colaborativo } \\
\text { El conocimiento se } \\
\text { construye }\end{array}$ & $\begin{array}{l}\text { Prefieren el trabajo } \\
\text { individual } \\
\text { El conocimiento sirve } \\
\text { para utilizarlo }\end{array}$ \\
\hline $\begin{array}{l}\text { Orientación de } \\
\text { aprendizaje }\end{array}$ & $\begin{array}{l}\text { Interés superficial } \\
\text { por el aprendizaje } \\
\text { No indagan en otras } \\
\text { fuentes, no realizan } \\
\text { todas las tareas }\end{array}$ & $\begin{array}{l}\text { Interés superficial } \\
\text { por el aprendizaje } \\
\text { No indagan en otras } \\
\text { fuentes } \\
\text { El aprendizaje se } \\
\text { orienta al logro de } \\
\text { una calificación }\end{array}$ & $\begin{array}{l}\text { Interés profundo por el } \\
\text { aprendizaje } \\
\text { Indagan en otras } \\
\text { fuentes } \\
\text { El aprendizaje se } \\
\text { orienta a adquirir } \\
\text { conocimientos }\end{array}$ & $\begin{array}{l}\text { Interés profundo por el } \\
\text { aprendizaje de los } \\
\text { contenidos con utilidad } \\
\text { práctica } \\
\text { Indagan en otras } \\
\text { fuentes } \\
\text { El aprendizaje se orienta } \\
\text { hacia la vocación }\end{array}$ \\
\hline
\end{tabular}

Este cambio se expresa en la impaciencia de los estudiantes cuando la información no se les presenta instantáneamente y en que no dedican mucho tiempo realizando una actividad. Esto desafía las conductas esperadas y las prácticas requeridas en el aula de las instituciones educativas: períodos de atención más largos y actividades reflexivas, entre otros (Ananiadou y Claro, 2009; Carstens y Beck, 2005). No obstante, Valtonen, et al. (2011) y Esteve y Gisbert (2013) enfatizan que no hay evidencia concluyente sobre esta caracterización de los NML, incluso afirman que la habilidad para el manejo de las TIC se enfoca para actividades sociales y lúdicas pero que muestran incapacidad para hacer la transferencia hacia el aprendizaje formal o al proceso de construcción del conocimiento. Una revisión sistemática sobre la literatura científica relacionada con los aprendices digitales, la realizaron Esteve et al. (2014), de los 344 artículos encontrados sobre esta temática, realizaron una síntesis sobre las características que diversos autores atribuyen a los NML, que se muestra en la tabla 3.

Tabla 3: Características del estudiante del nuevo milenio (Datos tomados de Esteve, Duch y Gisbert, 2014)

\begin{tabular}{|l|l|}
\hline Actitud ante las TIC & $\begin{array}{l}\text { Conectados permanentemente por medios electrónicos } \\
\text { Escasa capacidad para valorar relevancia de los contenidos digitales } \\
\text { Necesidad de utilizar las TIC con orientación multimedia }\end{array}$ \\
\hline Actitud personal & $\begin{array}{l}\text { Proactividad } \\
\text { Compromiso } \\
\text { Expresividad }\end{array}$ \\
\hline Patrones cognitivos & $\begin{array}{l}\text { Razonamiento no lineal, menor estructura mental } \\
\text { Estilo de aprendizaje visual } \\
\text { Atención discontinua } \\
\text { Sobrecarga cognitiva }\end{array}$ \\
\hline Actitud para el trabajo & $\begin{array}{l}\text { Gusto por el riesgo } \\
\text { Necesidad de gratificación instantánea } \\
\text { Independencia }\end{array}$ \\
\hline Actitudes sociales & $\begin{array}{l}\text { Mayor facilidad para establecer relaciones interpersonales digitalmente que físicamente } \\
\text { Inseguridad } \\
\text { Egocéntricos }\end{array}$ \\
\hline Actitudes educativas & $\begin{array}{l}\text { Preferencia por métodos educativos activos } \\
\text { Uso de las TIC como herramienta habitual } \\
\text { Facilidad de comunicación } \\
\text { Facilidad de acceso a la información }\end{array}$ \\
\hline
\end{tabular}


Todos los artículos referenciados abordan investigaciones realizadas principalmente en EEUU, Canadá, Inglaterra y Francia; encontrando un vacío en investigaciones que contribuyan a develar el perfil del estudiante del siglo XXI en países de América Latina, como México. Por lo anterior expuesto, se hace necesario que en todas las Instituciones de Educación Superior se realicen investigaciones dirigidas a analizar a los estudiantes para comprender ¿Cómo son? ¿Cómo aprenden? ¿Cómo estudian? ¿Cuál es el sentido que le dan a la escuela y a la educación superior? ¿Qué esperan de sus profesores?

Esta investigación se realizó en el Instituto Politécnico Nacional (IPN) que es una institución educativa mexicana que oferta 282 programas académicos, 51 para el Nivel Medio Superior, 81 para el Nivel Superior y 150 para Posgrado. Específicamente en el Nivel Superior dichos programas abarcan tres ramas de conocimiento: Ingeniería y Ciencias Físico Matemáticas (ICFM) (65\%), Ciencias Médico Biológicas (CMB) (24\%) y Ciencias Sociales y Administrativas (CSA) (11\%); para el año 2015 se tuvo con una matrícula de 104,439 alumnos distribuidos como se muestra en la tabla 4.

Tabla 4: Distribución de alumnos inscritos, en el 2015, del IPN

\begin{tabular}{|l|c|c|c|}
\hline Rama & Total & Hombres & Mujeres \\
\hline ICFM & 66,793 & $72 \%$ & $28 \%$ \\
\hline CMB & 17,123 & $39 \%$ & $61 \%$ \\
\hline CSA & 20,523 & $44 \%$ & $55 \%$ \\
\hline
\end{tabular}

El objetivo de la investigación fue describir el perfil de una muestra de estudiantes del nivel superior del IPN con base en sus necesidades de educación y sus expectativas sobre la educación superior y la práctica docente.

\section{MÉTODO}

La investigación se planteó como exploratoria - descriptiva (Hernández, et al., 2010), el estudio fue descriptivo transversal y la recolección de datos se efectuó de mayo a julio del 2015. Para lograr la validez en el diseño de esta investigación se cubrieron las siguientes pruebas (Babie, 2006): (i) validación del constructo porque las definiciones conceptuales de las categorías bajo estudio se desprendieron del marco teórico, (ii) validación externa estableciendo que los resultados que arroje la presente investigación se podrán generalizar solo para la muestra analizada y (iii) confiabilidad porque se desarrolló una bitácora para dejar constancia de los pasos seguidos, las técnicas y los instrumentos de recolección de la información utilizados, con los cuales se podría repetir la misma investigación para llegar a resultados similares.

El instrumento para la recolección de datos fue un cuestionario con 75 preguntas divididas en cinco categorías de análisis; la escala de medición se basó en la escala de Likert (Babie, 2006). Se realizó una prueba piloto para validar el cuestionario con 39 estudiantes inscritos en el segundo semestre del año escolar 2015 en dos sesiones de trabajo con una duración de 40 minutos cada una. En la primera sesión se les explicaron los objetivos de la investigación y los estudiantes resolvieron el cuestionario; en la segunda sesión se explicó detalladamente cada una de las categorías bajo estudio y se les solicitó retroalimentación sobre tres aspectos del cuestionario aplicado: 1) congruencia de las preguntas con la categoría analizada; b) claridad del lenguaje empleado y 3) valoración sobre la escala de medición. Los estudiantes señalaron la necesidad de ampliar la escala de las respuestas para algunas aseveraciones; dotar al cuestionario de instrucciones más claras y eliminar tres preguntas; ambas sesiones fueron grabadas. Después de analizar dichas grabaciones se reformuló el cuestionario. En la tabla 5 se describe el cuestionario aplicado y las definiciones operacionales de las categorías bajo estudio.

El método de muestreo fue no estadístico intencional, el criterio de inclusión fue ser un estudiante inscrito en Educación Superior del IPN y la participación fue voluntaria. Para el análisis de la información se utilizó el programa SPSS y se realizó en dos momentos, en el primero a partir de las respuestas de los estudiantes se estableció un perfil descriptivo con las cinco categorías de análisis; en el segundo se realizó un análisis por género centrando la discusión en las diferencias encontradas en las y los estudiantes. El cuestionario fue aplicado a un total de 497 estudiantes del IPN, de los cuales solo el $86 \%$ (considerado en adelante como el $100 \%$ ) contestó en su totalidad el cuestionario por lo que 68 cuestionarios fueron desechados. La edad de los estudiantes encuestados osciló entre 18 años a 30 años; $26 \%$ que cursaban cuarto semestre, $23 \%$ octavo, $18 \%$ primero, $9 \%$ tercero, $8 \%$ quinto, el porcentaje restante cursaba segundo y séptimo. Las características sociales de la muestra se pueden observar en la tabla 6. 
Tabla 5: Cuestionario de investigación

\begin{tabular}{|c|c|c|}
\hline Categoría de análisis & Subcategoría & Ítems \\
\hline \multirow[t]{2}{*}{ I. Educación Superior } & $\begin{array}{l}\text { a. Expectativas: Probabilidad de mejorar el nivel } \\
\text { de vida al terminar una carrera profesional. }\end{array}$ & 7 \\
\hline & $\begin{array}{l}\text { b. Formación: Integral (desarrollo armónico de } \\
\text { conocimientos, procedimientos, actitudes y } \\
\text { valores) versus profesional }\end{array}$ & 2 \\
\hline \multirow[t]{2}{*}{ II. Práctica docente } & $\begin{array}{l}\text { a. Características del buen docente: Cualidades } \\
\text { de un docente de calidad }\end{array}$ & 10 \\
\hline & $\begin{array}{l}\text { b. Buenas prácticas: Acciones y estrategias } \\
\text { utilizadas por el profesor para conducir el proceso } \\
\text { de enseñanza con eficacia }\end{array}$ & 7 \\
\hline \multicolumn{2}{|c|}{$\begin{array}{l}\text { III. Hábitos de estudio: Prácticas regulares que realizan los estudiantes para concretar el proceso de } \\
\text { aprendizaje. }\end{array}$} & 16 \\
\hline \multirow[t]{2}{*}{ IV. Formas de recibir y procesar información } & $\begin{array}{l}\text { a. Preferencia de formatos para la presentación } \\
\text { de contenidos o información: impreso versus } \\
\text { digital }\end{array}$ & 6 \\
\hline & $\begin{array}{l}\text { b. Estrategias de aprendizaje a partir de } \\
\text { información: prácticas que realizan los } \\
\text { estudiantes para asimilar, transformar y crear } \\
\text { conocimientos }\end{array}$ & 7 \\
\hline \multicolumn{2}{|c|}{$\begin{array}{l}\text { V. Actitudes ante el aprendizaje y desempeño profesional: Motivación de los estudiantes que se concreta } \\
\text { en proactividad por el aprendizaje y el desempeño profesional }\end{array}$} & 17 \\
\hline & Total & 72 \\
\hline
\end{tabular}

Tabla 6: Características sociales de la muestra

\begin{tabular}{|c|c|c|c|c|c|c|c|c|c|c|}
\hline & \multicolumn{5}{|c|}{ Hombres } & \multicolumn{5}{|c|}{ Mujeres } \\
\hline Encuestados & \multicolumn{5}{|c|}{$59 \%$} & \multicolumn{5}{|c|}{$41 \%$} \\
\hline Estado civil & \multicolumn{2}{|c|}{$\begin{array}{l}\text { Solteros } \\
96 \%\end{array}$} & \multicolumn{3}{|c|}{$\begin{array}{l}\text { Casados } \\
4 \%\end{array}$} & \multicolumn{2}{|c|}{$\begin{array}{l}\text { Solteras } \\
97 \%\end{array}$} & \multicolumn{3}{|c|}{$\begin{array}{l}\text { Casadas } \\
3 \%\end{array}$} \\
\hline Tienen hijos & \multicolumn{2}{|c|}{$\begin{array}{l}\text { si } \\
0 \%\end{array}$} & \multicolumn{3}{|c|}{$\begin{array}{l}\text { no } \\
100 \%\end{array}$} & \multicolumn{2}{|c|}{$\begin{array}{l}\mathrm{si} \\
4 \%\end{array}$} & \multicolumn{3}{|c|}{$\begin{array}{l}\text { no } \\
96 \%\end{array}$} \\
\hline Carrera que cursas & \multicolumn{2}{|c|}{$\begin{array}{l}\text { Ingeniería } \\
99 \%\end{array}$} & \multicolumn{3}{|c|}{$\begin{array}{l}\text { Licenciatura } \\
1 \%\end{array}$} & \multicolumn{2}{|c|}{$\begin{array}{l}\text { Ingeniería } \\
35 \%\end{array}$} & \multicolumn{3}{|c|}{$\begin{array}{l}\text { Licenciatura } \\
65 \%\end{array}$} \\
\hline $\begin{array}{l}\text { Computadora } \\
\text { personal }\end{array}$ & \multicolumn{2}{|c|}{$\begin{array}{l}\text { si } \\
96 \%\end{array}$} & \multicolumn{3}{|c|}{$\begin{array}{l}\text { no } \\
4 \%\end{array}$} & \multicolumn{2}{|c|}{$\begin{array}{l}\text { si } \\
95 \%\end{array}$} & \multicolumn{3}{|c|}{$\begin{array}{l}\text { no } \\
5 \%\end{array}$} \\
\hline Celular & \multicolumn{2}{|c|}{$\begin{array}{l}\text { si } \\
96 \%\end{array}$} & \multicolumn{3}{|c|}{$\begin{array}{l}\text { no } \\
4 \%\end{array}$} & \multicolumn{2}{|c|}{$\begin{array}{l}\text { si } \\
96 \%\end{array}$} & \multicolumn{3}{|c|}{$\begin{array}{l}\text { no } \\
4 \%\end{array}$} \\
\hline Tipo de celular & \multicolumn{2}{|c|}{$\begin{array}{l}\text { Smartphone } \\
80 \%\end{array}$} & \multicolumn{3}{|c|}{$\begin{array}{l}\text { Convencional } \\
20 \%\end{array}$} & \multicolumn{2}{|c|}{$\begin{array}{l}\text { Smartphone } \\
86 \%\end{array}$} & \multicolumn{3}{|c|}{$\begin{array}{l}\text { Convencional } \\
14 \%\end{array}$} \\
\hline $\begin{array}{l}\text { Nivel educativo del } \\
\text { padre }\end{array}$ & $\begin{array}{l}\text { Prim. } \\
9 \%\end{array}$ & $\begin{array}{l}\text { Sec. } \\
23 \%\end{array}$ & $\begin{array}{l}\text { Prep. } \\
34 \%\end{array}$ & $\begin{array}{l}\text { Lic. } \\
31 \%\end{array}$ & $\begin{array}{l}\text { Posgrado } \\
3 \%\end{array}$ & $\begin{array}{l}\text { Prim. } \\
10 \%\end{array}$ & $\begin{array}{l}\text { Sec. } \\
30 \%\end{array}$ & $\begin{array}{l}\text { Prep. } \\
27 \%\end{array}$ & $\begin{array}{l}\text { Lic. } \\
30 \%\end{array}$ & $\begin{array}{l}\text { Posgrado } \\
3 \%\end{array}$ \\
\hline $\begin{array}{l}\text { Nivel educativo de } \\
\text { la madre }\end{array}$ & $\begin{array}{l}\text { Prim. } \\
11 \%\end{array}$ & $\begin{array}{l}\text { Sec. } \\
26 \%\end{array}$ & $\begin{array}{l}\text { Prep. } \\
32 \%\end{array}$ & $\begin{array}{l}\text { Lic. } \\
28 \%\end{array}$ & $\begin{array}{l}\text { Posgrado } \\
3 \%\end{array}$ & $\begin{array}{l}\text { Prim. } \\
16 \%\end{array}$ & $\begin{array}{l}\text { Sec. } \\
27 \%\end{array}$ & $\begin{array}{l}\text { Prep. } \\
36 \%\end{array}$ & $\begin{array}{l}\text { Lic. } \\
17 \%\end{array}$ & $\begin{array}{l}\text { Posgrado } \\
4 \%\end{array}$ \\
\hline
\end{tabular}

La carrera de los estudiantes encuestados fue del 52\% Ingeniería en Sistemas Computacionales, del $18 \%$ Licenciatura en Turismo, del 15\% Ingeniería en Informática, del 6\% Ingeniería Civil, del 4\% Licenciatura en 
Administración Industrial y del restante $5 \%$ otras licenciaturas.

\section{RESULTADOS Y DISCUSIÓN}

Tomando en cuenta la naturaleza exploratoria - descriptiva de la investigación los resultados presentados corresponden a la muestra analizada, para poder hacer una generalización estadística se requiere sumar estos resultados a otras investigaciones en diversos contextos. Este apartado se divide en cada una de las cinco categorías de análisis, lo que permitirá más adelante realizar una descripción genérica del perfil de la muestra de estudiantes del IPN encuestados.

\section{Educación superior}

De manera general se puede apreciar una percepción homogénea, en los estudiantes encuestados, sobre la importancia de la educación superior para lograr éxito laboral, mostrando preferencia por el autoempleo y la formación empresarial tal como se muestra en la tabla 7.

Tabla 7: Educación superior

\begin{tabular}{|c|c|c|c|c|c|}
\hline ¿Qué tan de acuerdo o en desacuerdo estás con las siguientes aseveraciones? & $T A$ & $D A$ & NES & $E D$ & $T D$ \\
\hline $\begin{array}{l}\text { La educación superior me proporcionará las herramientas necesarias para } \\
\text { triunfar en mi campo laboral }\end{array}$ & \multicolumn{2}{|c|}{$82 \%$} & $16 \%$ & & \\
\hline El prestigio de la escuela me motivó a elegirla & $87 \%$ & & & \multicolumn{2}{|c|}{$13 \%$} \\
\hline $\begin{array}{l}\text { La formación profesional es más importante que la investigación y la extensión } \\
\text { (cultura, deporte) }\end{array}$ & \multicolumn{2}{|c|}{$71 \%$} & & \multicolumn{2}{|c|}{$29 \%$} \\
\hline $\begin{array}{l}\text { La escuela debe atender las demandas de las empresas para formarnos con } \\
\text { calidad }\end{array}$ & $89 \%$ & & & \multicolumn{2}{|c|}{$11 \%$} \\
\hline Prefiero que me formen para el empleo que para generar empleos & \multicolumn{2}{|c|}{$32 \%$} & & \multicolumn{2}{|c|}{$68 \%$} \\
\hline $\begin{array}{l}\text { El establecimiento de convenios empresa-escuela contribuyen a mejorar mi } \\
\text { formación profesional }\end{array}$ & $93 \%$ & & & $7 \%$ & \\
\hline
\end{tabular}

Es importante enfatizar que a pesar de los esfuerzos de la UNESCO (1998), la OEI (2010) y la ANUIES (2000) para que en las instituciones de educación superior se ofrezca una formación integral, 7 de cada diez estudiantes encuestados consideren más importante la formación profesional que el desarrollo de competencias de investigación o el fomento de actividades culturales y deportivas. Esto podría impactar con la aspiración del aprendizaje permanente para los estudiantes (UNESCO, 2007) sí no se propicia en ellos la necesidad de mantenerse vigentes en su respectivo campo laboral.

\section{Práctica docente}

Se les preguntó sobre las cualidades que consideraban que los buenos docentes deberían de manifestar durante la conducción del proceso de enseñanza - aprendizaje. Los resultados se muestran en la tabla 8.

Tabla 8: Características de un buen docente

\begin{tabular}{|l|c|}
\hline & Menciones \\
\hline $\begin{array}{l}\text { CONGRUENCIA Desean que los docentes establezcan el mismo nivel de exigencia sobre su } \\
\text { desempeño }\end{array}$ & 273 \\
\hline ACTUALIZACIÓN Que estén actualizados en el manejo de tecnologías e innovación & 229 \\
\hline MOTIVACIÓN Esperan motivación para aprender y retroalimentación en tiempo y forma & 223 \\
\hline COMPROMISO Se muestren comprometidos con el aprendizaje de todo el grupo & 215 \\
\hline $\begin{array}{l}\text { RESPETO Los estudiantes prefieren que se les llame por su nombre y que no se burlen de las fallas } \\
\text { que pudieran tener durante el ciclo escolar }\end{array}$ & 184 \\
\hline $\begin{array}{l}\text { ORGANIZACIÓN Preparen y organicen sus clases con anticipación; así como que lleven orden en las } \\
\text { evaluaciones y en la revisión de tareas y actividades }\end{array}$ & 168 \\
\hline NO AUTORITARISMO Les molesta que el docente utilice su posición para demostrar poder y valor & 223 \\
\hline
\end{tabular}




\begin{tabular}{|l|c|}
\hline BUEN HUMOR Prefieren un docente que establezca un buen clima de clase & 165 \\
\hline RESPONSABILIDAD Que asistan con puntualidad y cumplan con lo prometido & 163 \\
\hline
\end{tabular}

Como se observa, los estudiantes encuestados manifiestan como cualidades importantes la congruencia y actualización, es decir competencias cognitivas, y éticas (Osuna y Luna, 2008); llama la atención que presenten una motivación externa para el aprendizaje y requieran de sus docentes amplia retroalimentación de su desempeño o de sus detenciones en el aprendizaje casi en tiempo real (menos de 24 horas); práctica que actualmente no siguen los docentes. Los docentes se encuentran ante estudiantes altamente dependientes y demandantes lo que va en contrasentido de lo esperado por la Tercera Reforma Educativa para América Latina (UNESCO, 2003) que pretende que los estudiantes se responsabilicen por su propio aprendizaje. Por otro lado, de acuerdo con Monroy y Hernández (2014), Baerten, et al. (2010) y Cornejo y Arredondo (2007) la motivación extrínseca se relaciona con un aprendizaje superficial caracterizada por un procesamiento dirigido a la reproducción (Vermunt, 1996).

Con el objetivo de hacer visibles los patrones o estrategias de enseñanza que los estudiantes encuestados consideran de mayor valor para aprender se les preguntó a los estudiantes por las buenas prácticas docentes (Zabalza, 2002); el $91 \%$ concuerda en que una buena práctica es resolver todas las dudas que surjan y que les explique paso a paso todos los contenidos. Asimismo, el $77 \%$ considera a la disciplina dentro del aula como un factor importante para favorecer el proceso de enseñanza - aprendizaje; y el $87 \%$ de los respondientes consideran que el hacer las clases interesantes está fuertemente relacionado con la calidad del desempeño de un docente. Estos resultados nos indican que los estudiantes prefieren un modelo de enseñanza basado en el docente ya que ellos prefieren desempeñar un rol secundario (Gimeno y Pérez, 2005).

\section{Hábitos de estudio}

Los resultados obtenidos sobre los hábitos de estudio de los estudiantes encuestados muestran menor homogeneidad en su conjunto. La mayoría de las respuestas a las preguntas realizadas se concentraron en el frecuentemente y ocasionalmente, como se muestra en la tabla 9. Resalta que la mayoría de los estudiantes encuestados no establece horarios para estudiar y no llevan a cabo una planeación con la programación de sus actividades por lo que no regulan su proceso de aprendizaje (Covey, 2012; Vermunt, 1996). Resalta que aunque afirman fijarse metas y objetivos de aprendizaje, el $64 \%$ no elabore un plan para dichos objetivos. El tiempo invertido diariamente para realizar sus actividades o estudiar es muy variado. El $9 \%$ manifiesta invertir un promedio de tres o más horas; el 19\% de dos a tres horas diarias; el $37 \%$ de una a dos horas diariamente en tanto que el $34 \%$ no dedica tiempo o invierte menos de una hora al día.

Tabla 9: Hábitos de estudio

\begin{tabular}{|c|c|c|c|c|}
\hline ¿Con qué regularidad realizas estas actividades? & Siempre & Frecuentemente & Ocasionalmente & $\begin{array}{l}\text { Rara vez } \\
\text { / Nunca }\end{array}$ \\
\hline Estableces horarios para estudiar & $45 \%$ & & & $55 \%$ \\
\hline $\begin{array}{l}\text { Cuando estudias te concentras solamente en realizar } \\
\text { esta actividad }\end{array}$ & \multicolumn{2}{|r|}{$8 \%$} & \multicolumn{2}{|l|}{$92 \%$} \\
\hline Realizas todas tus actividades / tareas académicas & \multicolumn{2}{|r|}{$76 \%$} & & $24 \%$ \\
\hline Elaboras un plan de trabajo y lo cumples & \multicolumn{2}{|r|}{$36 \%$} & $64 \%$ & \\
\hline Te fijas objetivos o metas de aprendizaje & $36 \%$ & \multicolumn{2}{|c|}{$61 \%$} & \\
\hline $\begin{array}{l}\text { Aprovechas tus traslados (casa - escuela) para } \\
\text { estudiar con tu teléfono celular }\end{array}$ & $10 \%$ & $24 \%$ & \multicolumn{2}{|l|}{$66 \%$} \\
\hline $\begin{array}{l}\text { Utilizas alguna aplicación (móvil o de computadora) } \\
\text { que te ayude a organizar tus estudios }\end{array}$ & & $11 \%$ & $30 \%$ & $59 \%$ \\
\hline $\begin{array}{l}\text { Utilizas mensajería instantánea para comunicarte con } \\
\text { tus compañeros y resolver dudas de las asignaturas } \\
\text { o de las tareas }\end{array}$ & \multicolumn{2}{|r|}{$67 \%$} & \multicolumn{2}{|l|}{$33 \%$} \\
\hline $\begin{array}{l}\text { Utilizas mensajería instantánea para comunicarte con } \\
\text { tus profesores para preguntar sobre dudas de las } \\
\text { asignaturas o de las tareas }\end{array}$ & \multicolumn{2}{|r|}{$22 \%$} & \multicolumn{2}{|l|}{$78 \%$} \\
\hline
\end{tabular}

El teléfono celular, la computadora, la música y la televisión están muy presentes cuando de estudiar se trata; el $92 \%$ de los estudiantes encuestados afirman no apagar su celular mientras están estudiando y el $64 \%$ enfatiza que también escuchan música, ven televisión e interactúan en redes sociales. Esto podría 
relacionarse con los resultados de Esteve, et al. (2014) sobre la atención discontinua y la sobrecarga cognitiva que presentan los estudiantes y que les podría estar obstaculizando obtener mejores resultados en la escuela, solo el $68 \%$ de los encuestados eran alumnos regulares (sin adeudos de materias). 
En los últimos años el uso de mensajeros instantáneos (Skype, WhatsApp) se ha intensificado, el 67\% utilizan estos medios para contactar a sus compañeros fuera de la escuela y resolver alguna duda y el $22 \%$ de los encuestados, para ese fin y por esos mismos medios, también contacta a sus maestros, aunque el $50 \%$ se muestra insatisfecho con el tiempo que tardan los docentes en responder, en promedio dos días.

\section{Formas de recibir y procesar la información}

Los estudiantes se encuentran permanentemente conectados y reciben información continuamente, aunque no la utilizan para la construcción del conocimiento (Esteve y Gisbert, 2013) como muestran los resultados mostrados en la tabla 10.

Tabla 10: Formas de recibir y procesar la información

\begin{tabular}{|c|c|c|c|c|}
\hline $\begin{array}{l}\text { Al momento de estudiar o realizar tus actividades } \\
\text { académicas... }\end{array}$ & Siempre & Frecuentemente & Ocasionalmente & $\begin{array}{l}\text { Rara } \\
\text { vez / } \\
\text { Nunca }\end{array}$ \\
\hline $\begin{array}{l}\text { ¿Utilizas información obtenida en tus redes sociales } \\
\text { (Facebook, Twitter, u otro)? }\end{array}$ & & $42 \%$ & \multicolumn{2}{|l|}{$58 \%$} \\
\hline $\begin{array}{l}\text { ¿Prefieres los libros electrónicos que los textos en } \\
\text { papel? }\end{array}$ & & $35 \%$ & \multicolumn{2}{|l|}{$65 \%$} \\
\hline $\begin{array}{l}\text { ¿Lees las lecturas completas que te mandan tus } \\
\text { profesores? }\end{array}$ & & $27 \%$ & \multicolumn{2}{|l|}{$73 \%$} \\
\hline $\begin{array}{lcll}\text { ¿Acostumbras } & \text { complementar } & \text { tu } & \text { aprendizaje } \\
\text { buscando información adicional? } & & \end{array}$ & & $60 \%$ & \multicolumn{2}{|l|}{$40 \%$} \\
\hline ¿Imprimes las lecturas digitales? & \multicolumn{2}{|r|}{$22 \%$} & $78 \%$ & \\
\hline ¿Acostumbras tomar notas de lo expuesto en clase? & $55 \%$ & $30 \%$ & $15 \%$ & \\
\hline $\begin{array}{llllll}\text { ¿Acostumbras realizar una } & \text { síntesis } & \text { de } & \text { la } \\
\text { información? }\end{array}$ & $13 \%$ & \multicolumn{2}{|c|}{$74 \%$} & $13 \%$ \\
\hline ¿Acostumbras subrayar o resaltar los textos? & \multicolumn{2}{|r|}{$75 \%$} & \multicolumn{2}{|l|}{$25 \%$} \\
\hline $\begin{array}{l}\text { Para libros electrónicos ¿utilizas alguna aplicación } \\
\text { (móvil o de computadora) que te permita tomas notas } \\
\text { y/o resaltar información? }\end{array}$ & & $15 \%$ & & $85 \%$ \\
\hline $\begin{array}{l}\text { Prefiero ir a lo concreto y no perder el tiempo leyendo } \\
\text { contenidos extensos }\end{array}$ & \multicolumn{2}{|r|}{$78 \%$} & \multicolumn{2}{|l|}{$22 \%$} \\
\hline Esquivo los temas subjetivos o poco claros & \multicolumn{2}{|r|}{$55 \%$} & \multicolumn{2}{|l|}{$45 \%$} \\
\hline ¿Te agrada el trabajo metódico? & \multicolumn{2}{|r|}{$21 \%$} & \multicolumn{2}{|l|}{$79 \%$} \\
\hline \multicolumn{5}{|l|}{ De las siguientes opciones elige cuál prefieres: } \\
\hline a) Discutir temas & \multicolumn{2}{|r|}{$64 \%$} & \multicolumn{2}{|l|}{$36 \%$} \\
\hline b) Explicación detallada de temas & \multicolumn{3}{|c|}{$36 \%$} & $64 \%$ \\
\hline
\end{tabular}

Resalta el hecho de que los alumnos encuestados prefieran los textos en papel a los libros electrónicos, esto nos habla de que la incorporación tecnológica aún no extiende plenamente a la educación; esto se refuerza con la afirmación de no utilizar aplicaciones que les permitan tomar notas o resaltar partes importantes de los textos consultados. Seis de cada diez estudiantes afirman ser proactivos y buscar información adicional a los temas expuestos por el docente aunque manifiestan que no para todos los temas, únicamente para aquellos temas que consideran que les servirán para su profesión y el formato preferido es visual al buscar tutoriales y videos (Oblinger, 2005; Carstens y Beck, 2005). Los resultados parecen confirmar que los estudiantes encuestados presentan un procesamiento cognitivo concreto y mayormente están interesados en aprendizajes de utilidad práctica (Vermunt, 1996); prefieren que se le dé información concreta más que de carácter general y que se les diga, sin mayor preámbulo, lo que necesitan saber o conocer. El 64\% prefiere discutir temas antes que las explicaciones detalladas.

\section{Actitudes ante el aprendizaje y el desempeño profesional}

Las actitudes ante el aprendizaje, que influyen en el rendimiento académico de los estudiantes, y el desempeño profesional, que les permite anticipar lo que van a requerir para su futuro trabajo, se consideraron elementos importante para la descripción del perfil de la muestra de estudiantes. Ante la 
pregunta de qué es el aprendizaje el 50\% manifestó que aprender es aplicar conocimientos para resolver problemas, el $41 \%$ consideró que era comprender nuevas cosas que dan sentido a los saberes previos; adicionalmente, se les preguntó cuándo se dan cuenta de que aprendieron, el $46 \%$ expresó que cuando se lo pueden explicar a otras personas, el 30\% manifestó que cuando resuelven problemas y ejercicios sin dificultad y el $15 \%$ respondió que cuando integran lo aprendido a lo que ya sabían. Estas características coinciden con un estilo de aprendizaje dirigido a la aplicación de lo aprendido (Vermunt, 1996).

Aunque los estudiantes encuestados prefieren discutir temas, al $88 \%$ no le gusta participar en las mismas y prefieren escuchar la discusión; igual pasa con el trabajo en equipo aunque en menor porcentaje de aceptación, el $57 \%$ prefiere el trabajo en equipo para los proyectos escolares pero al $80 \%$ de los mismos no les gusta el rol de liderazgo en los equipos. Considerando que el IPN persigue, en sus diferentes perfiles de egreso, que los egresados sean capaces de liderar equipos de trabajo multidisciplinarios (IPN, 2010) la actitud mostrada por los encuestados muestra un área de oportunidad que deberá atenderse.

Otras características relacionadas con la actitud ante el aprendizaje fueron el autoconcepto y la motivación para aprender, en la tabla 11 se presentan estos resultados.

Tabla 11: Autoconcepto y motivación de los estudiantes

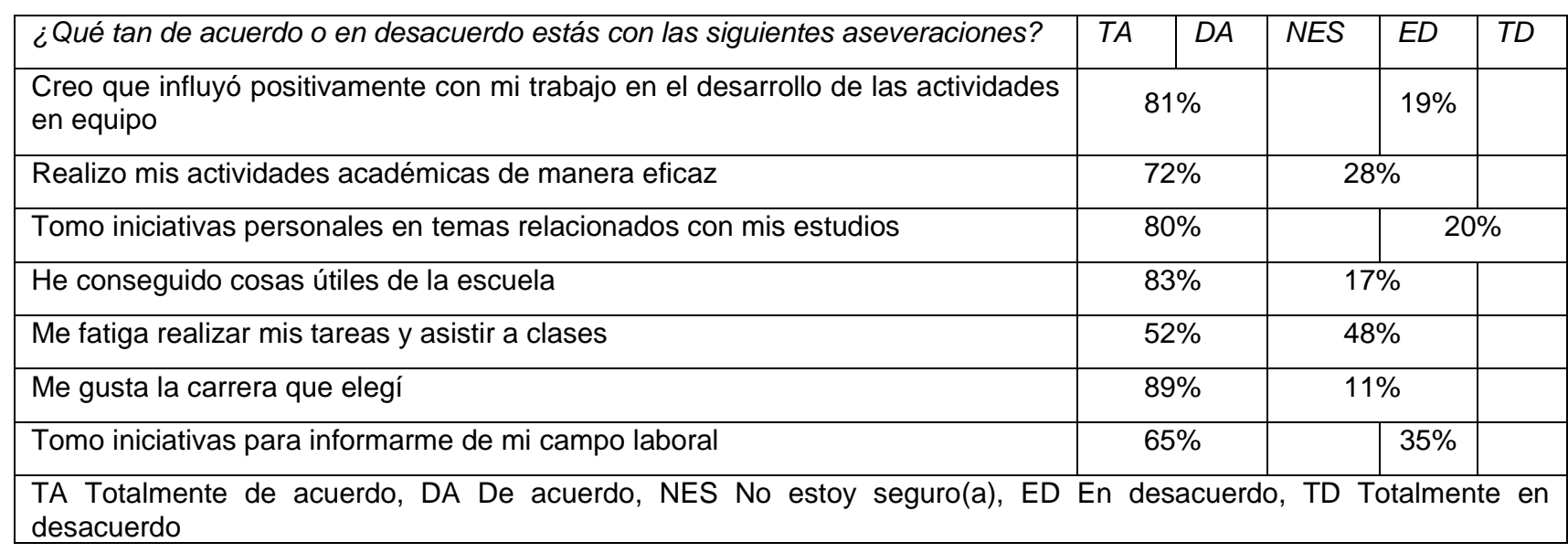

Los estudiantes encuestados afirman ser proactivos y tomar decisiones relacionadas con su formación. Por otro lado, ocho de cada diez estudiantes manifiestan con seguridad influir positivamente en sus equipos de trabajo y se perciben como eficaces en la realización de sus actividades académicas, estos resultados no concuerdan con la investigación realizada por Esteve et al. (2014) relacionada con las actitudes sociales de los estudiantes del nuevo milenio.

\section{Perfil de la muestra de estudiantes del Instituto Politécnico Nacional}

El perfil describe las expectativas que la muestra de estudiantes del IPN tienen sobre la educación superior, la práctica docente, sus hábitos de estudio, sus preferencias en cuanto a las formas de recibir y procesar información y actitudes frente al aprendizaje y el desempeño profesional.

Como se observa en la tabla 12, tienen altas expectativas sobre la educación superior por lo que investigan y eligen dónde van a hacerlo. Esperan una formación más profesional que integral y los acuerdos de vinculación escuela - empresa son vistos como convenientes pues les permitirán formarse mejor. Asimismo, las expectativas que tienen de sus docentes corresponden a un perfil más carismático y menos autoritario, desean retroalimentación puntual sobre sus dudas y su desempeño; esperan clases interesantes y el que el desarrollo de los contenidos sea concreto. No presentan una actitud estratégica sobre sus estudios aunque 6 de cada diez si la tiene sobre su campo profesional; explotan altamente las TIC con fines interactivos y de entretenimiento, pero no se apoyan en ellas para establecer estrategias de aprendizaje.

\section{CONCLUSIONES}

Con base en el análisis, la discusión y los resultados obtenidos, se pueden obtener las siguiente conclusiones principales: 1) los estudiantes están permanentemente conectados a la red, aunque el dominio de la tecnología digital no está incorporado a sus actividades académicas; 2 ) un hallazgo relevante es que prefieren los textos en papel para estudiar; 3) esperan que sus docentes retroalimenten su desempeño o aclaren sus dudas, manifestadas por correo electrónico, en menos de 24 horas; 4) presentan una orientación hacia el aprendizaje práctico y concreto, son proactivos y buscan información adicional 
solamente para contenidos de utilidad práctica; 5) exteriorizan una autoestima alta y están seguros de haber logrado grandes cosas en su vida académica, independientemente de su promedio escolar; y 6) prefieren como estrategia de enseñanza la discusión de temas, pero no participan activamente en ellas, y la elaboración de proyectos en equipos de trabajo pero a ocho de cada diez no les gusta ser los líderes de los mismos. Esto último representa un área de oportunidad porque el IPN persigue, en sus diferentes perfiles de egreso, que los egresados sean capaces de liderar equipos de trabajo multidisciplinarios.

Tabla 12: Perfil de la muestra de estudiantes analizada

\begin{tabular}{|c|c|}
\hline $\begin{array}{l}\text { Educación } \\
\text { Superior }\end{array}$ & $\begin{array}{l}\text { Consideran la Educación Superior les permitirá triunfar en el campo laboral } \\
\text { Siguen un proceso deliberado al momento de elegir dónde cursar su carrera profesional } \\
\text { Prefieren recibir una formación profesional centrada en atender las demandas del mercado laboral } \\
\text { Consideran que la vinculación entre la escuela y las empresas es conveniente para su formación } \\
\text { profesional }\end{array}$ \\
\hline $\begin{array}{l}\text { Práctica } \\
\text { docente }\end{array}$ & $\begin{array}{l}\text { Valoran de sus docentes la competencia cognitiva - técnica y la ética } \\
\text { Requieren retroalimentación constante } \\
\text { Esperan que el docente mantenga la disciplina dentro del aula } \\
\text { Prefieren más discutir temas que las explicaciones de los mismos } \\
\text { Prefieren como estrategias de enseñanza el desarrollo de proyectos y los debates en clase }\end{array}$ \\
\hline $\begin{array}{l}\text { Hábitos de } \\
\text { estudio }\end{array}$ & $\begin{array}{l}\text { No establecen horarios para estudiar sus clases } \\
\text { Escasa autorregulación del proceso de aprendizaje. Solo planean entregas de proyectos y } \\
\text { realización de exámenes } \\
\text { No utilizan aplicaciones para organizar actividades académicas } \\
\text { Cuando estudian acostumbran ver televisión u oír música, interactuar en sus redes sociales y } \\
\text { atender su celular }\end{array}$ \\
\hline $\begin{array}{l}\text { Formas de } \\
\text { recibir y } \\
\text { procesar } \\
\text { información }\end{array}$ & $\begin{array}{l}\text { Prefieren información presentada en formatos multimedia } \\
\text { Prefieren estudiar libros de texto antes que en libros electrónicos } \\
\text { Explotan altamente las TIC, aunque no para establecer estrategias de aprendizaje } \\
\text { No acostumbran hacer una síntesis de la información } \\
\text { Procesamiento de información concreto }\end{array}$ \\
\hline $\begin{array}{l}\text { Actitudes ante } \\
\text { el aprendizaje } \\
\text { y desempeño } \\
\text { profesional }\end{array}$ & $\begin{array}{l}\text { Orientación del aprendizaje dirigido hacia la aplicación } \\
\text { Buscan información adicional solo para contenidos de utilidad práctica } \\
\text { Consideran que han aprendido algo cuando son capaces de explicárselo a otras personas } \\
\text { No les agrada ser líderes de equipo } \\
\text { Les disgusta el trabajo minucioso y/o metódico } \\
\text { Presentan autoestima y un buen autoconcepto } \\
\text { Se mantienen informados sobre su futuro campo profesional }\end{array}$ \\
\hline
\end{tabular}

\section{AGRADECIMIENTOS}

Los autores agradecen al Instituto Politécnico Nacional las facilidades prestadas para la realización de esta investigación.

\section{REFERENCIAS}

Alonso, C. y D. Gallego, Estilos de aprendizaje: Teoría y práctica, UNED, Madrid, España (2004)

Ananiadou, K. y Claro, M., $21^{\text {ST }}$ Century Skills and Competences for new Millennium Learners in OECD countries, doi: 10.1787/19939019, OECD Education Working Papers, 41, 1-34 (2009)

ANUIES, La educación superior del siglo XXI, 1aㅡ., ANUIES, Ciudad de México, México (2000)

Argudín, Y., Educación Basada en Competencias. Nociones y antecedentes, Trillas, Ciudad de México, 
México (2005)

Babie, E., Fundamentos de la investigación social, Thomson, Distrito Federal, México (2006)

Baerten, M., E. Kyndt, K. Struyven, y F. Dochy, Using student-centred learning enviroments to stimulate deep approaches to learning: Factors encouranging or discouranging their effectiveness, https://goo.gl/msZqNr, ISSN: 1747938X, Educational Research Journal, 5(3), 243-260 (2010)

Beca, C., C. E. García, P. Montt, C. Sotomayor y H. Walker, Docentes para el Nuevo Siglo. Hacia una Política de Desarrollo Profesional Docente, Ministerio de Educación, Dirección de comunicaciones, Santiago de Chile (2006)

Cano, E., La evaluación por competencias en la educación superior, https://goo.gl/rTV4ZZ, ISSN: 1989639X, Currículum y Formación del Profesorado, 3(12), 1-16 (2008)

Carreras, J. y P. Perrenoud, El debate sobre las competencias en la enseñanza universitaria, Octaedro, Barcelona, España (2008)

Carstens, A. y Beck, J., Get Ready for the Gamer Generation, doi: 10.1007/BF02763643, TechTrends, 49(3), 22-25 (2005), (por suscripción), https://goo.gl/1PJAuf (2005)

Cornejo, R., M. Redondo, Variables y factores asociados al aprendizaje escolar. Una discusión desde la investigación actual, https://goo.gl/ZVGZoX, ISSN: 0718-0705, Estudios Pedagógicos XXXIII (2), 155-177 (2007)

Covey, S., Los siete hábitos de los adolescentes altamente efectivos, Debolsillo, Madrid, España (2012)

Díaz-Barriga, F. y L. Hernández, Estrategias docentes para un aprendizaje significativo. Una interpretación constructivista, Mc Graw Hill, Distrito Federal, México (2002)

Díaz Barriga, F., Enseñanza situada. Vínculo para la escuela y la vida, Mc Graw Hill, Ciudad de México, México (2006)

Dubet, F., ¿Mutaciones institucionales y/o neoliberalismo?, en Gobernabilidad de los sistemas educativos en América Latina por E. T. Fanfani, IIPE UNESCO, 15-43, Buenos Aires, Argentina (2004)

Esteve, F. y M. Gisbert, Competencia digital y nuevos entornos: Instrumentos de evaluación y nuevos entornos, https://goo.gl/SrmWTQ, ISSN: 1690-7515 Enl@ce: Revista Venezolana de Información, Tecnología y Conocimiento 10(3), 29-43 (2013)

Esteve, F., J. Duch, y M. Gisbert, Los aprendices digitales en la literatura científica: Diseño y aplicación de una revisión sistemática entre 2001 y 2010, https://goo.gl/KsD4JJ, ISSN: 1133-8482, Revista de Medios y Educación, 45, 9-21 (2014)

Fullan, M., The New meaning of educational change, 5ㄹ Ed., 18-38, Teachers College Press, New York, USA (2015)

Gimeno, J. y Á. Pérez, Comprender y transformar la enseñanza, Morata, Madrid, España (2005)

Gorodokin, I., La formación docente y su relación con la epistemología, http://rieoei.org/1164.htm, ISSN: 1681-5653, Revista Iberoamericana de Educación, 35, 1-9 (2005)

Hernández, R., C. Fernández y M. Baptista, Metodología de la investigación, 5aㅡ Ed., Mc Graw Hill, Distrito Federal, México (2010)

Imbernón, F., Un nuevo profesorado para una nueva universidad. ¿Conciencia o presión?, Red Interuniversitaria de Formación del Profesorado, https://goo.gl/6TBiEC, ISSN: 0213-8646, 38, 37-46 (2000)

Jiménez, Y., J. Hernández y D. Ortega, Forman los programas de formación docente, https://goo.gl/oiCKrg, ISSN: 18705308, CPU-e Investigación Educativa, 19, 1-27 (2014)

Marchesi, A., Metas Educativas 2021: un proyecto iberoamericano para transformar la educación en la década de los bicentenarios, https://goo.gl/oK2KJn, ISSN: 1850-0013, Revista Iberoamericana de Ciencia Tecnología y Sociedad 4(12), 87-156 (2009) 
Monroy, F. y F. Hernández, Factores que influyen en el aprendizaje universitario. Una revisión sistémica, doi: 10.5944/eduexx1.17.2.11481, Educación XXI, 17(2), 105-124 (2014)

Morán, L., Criterios para análisis comparativo de modelos y diseños educativos, https://goo.gl/9eAsZ7, ISSN: 0123-1294, Educación y educadores, 11(2), 139-158 (2008)

Oblinger, D., Is the age or IT: First steps understanding the next generation in Educating the Net Generation by D. Oblinger y J. Oblinger (Ed.) Educause, 2.1-2.20, https://goo.gl/qxvvqt, Washington DC, USA (2005)

OEI, 2021 Metas educativas. La educación que queremos para la generación de los bicentenarios. Documento final (en la web: https://goo.gl/x7RSxd, acceso: 15 de marzo 2015), ISBN: 978-84-7666-224-3, Depósito Legal: M-36970-2010, Madrid, España (2010)

Osuna, C. y E. Luna, Características de ser un buen profesional de Ingeniería en la Universidad Autónoma de Baja California, México, doi: 10.4067/S0718-50062008000500005, Formación Universitaria 1(1), 29-36 (2008)

Pedró, F., The new millenium learners: Challenging our views on ICT and learning, (en la web: https://goo.gl/MTMPWa, acceso: 16 de abril 2015), OECD - CERI, París, Francia (2006)

Prawat, R., Teachers' beliefs about teaching and learning: A constructivist perspective, doi: 10.1086/444021, American Journal of Education, 5(1), 354-395 (1992)

Rama, C., La tercera reforma educativa en América Latina, Fondo de Cultura Económica, Buenos Aires, Argentina (2006)

Retana, J.A., Modelo educativo basado en competencias: importancia y necesidad, https://goo.gl/w33ntK, ISSN: 1409-4703, Actualidades investigativas en educación, 11(3), 1-24 (2011).

Ruiz, M., El proceso curricular por competencias, Trillas, Distrito Federal, México (2009)

UNESCO, Higher education in the Twenty - first Century. Vision and action, UNESCO, París, Francia (1998)

UNESCO, Tendencias de la educación superior en América Latina y el Caribe, CRESALC/UNESCO, Caracas, Venezuela (2003)

UNESCO, Education for All. The quality imperative, UNESCO, París, Francia (2004)

UNESCO, Educación de calidad para todos: Un asunto de derechos humanos, UNESCO, Buenos Aires, Argentina (2007)

Valtonen, T., S. Pontinen, J. KukKonen, P. Dillon, P. Vaisanen y H. Stina, Confronting the technological pedagogical knowledge of Finnish Net Generation student teachers, https://goo.gl/MLZ4Tt, Technology, Pedagogy and Education, 20(1), 3-18 (2011)

Vermunt, J. D., Metacognitive, cognitive and affective aspects of learning styles. A phenomenographic analysis, doi: 10.1007/BF00129106, Higher Education, 31(1), 25-50 (1996)

Windschitl, M., Framing Constructivism in Practice as the Negotiation of Dilemmas: An Analysis of the Conceptual, Pedagogical, Cultural and Political Challenges Facing Teachers, doi: 10.3102/00346543072002131, Review of Educational Research, 72, 131-175 (2002)

Zabala A. y L. Arnau, El aprendizaje y la enseñanza por competencias. Colec. 11 ideas clave para educar, Graó, Barcelona, España (2007)

Zabalza, M., La enseñanza universitaria. El escenario y sus protagonistas, Narcea, Madrid, España (2002)

Zembylas, M., Teachers emotions of educational reform, En: Second International Handbook of educational change, pp. 221-236. Editores: Andy Hargreaves, Ann Lieberman, Michael Fullan y David Hopkins. Springer, New York, USA (2010) 
